\title{
Bioimpedancia: herramienta habitual en los cuidados de los pacientes de diálisis peritoneal (DP)
}

"Premio Fresenius Medical Care para enfermería nefrológica. Diálisis Peritoneal 2010”

Gema Vinagre Rea, Patricia Arribas Cobo, Irene Callejo Cano, Mª Amor Martínez Aranda, Sonia García Estévez

Enfermeras Hospital Universitario Infanta Leonor. Madrid

\section{Resumen}

El estado de hidratación de los pacientes debe ser considerado como un indicador de diálisis adecuada, además de influir directamente en la calidad de vida del paciente.

La Bioimpedancia es un método de valoración objetiva y de monitorización nutricional e hídrica de los pacientes. Las mediciones periódicas pueden ser complemento útil para la valoración clínica y la identificación de variaciones en la hidratación y nutrición, permitiendo alteraciones adecuadas de la conducta.

En nuestra unidad, nos planteamos utilizar la bioimpedancia como herramienta para detectar el estado de hidratación y la composición corporal de los pacientes para elaborar un plan de cuidados individualizado.

Se realizó en nuestro centro un estudio analizando en una muestra de 8 pacientes en programa de diálisis peritoneal, las mediciones por bioimpe-

\section{Correspondencia:}

Gema Vinagre Rea

Hospital Universitario Infanta Leonor

Servicio de Nefrología

Avenida Gran Vía del Este 80

28038 Madrid.

e-mail: gmaria.vinagre@salud.madrid.org dancia que de forma rutinaria se realizaron en cada revisión.

Las variables recogidas fueron edad, causa de enfermedad renal (ER), peso, altura, sexo, tiempo en diálisis, presión arterial (PA), filtrado glomeru$\operatorname{lar}(F G), n^{\circ}$ de hipotensores, diuréticos y resultados obtenidos de las mediciones de bioimpedancia.

Podemos concluir que la bioimpedancia detecta pacientes con hipertensión no dependiente de volumen. En estos casos es fundamental no forzar el aumento de UF para disminuir la PA, puesto que esto afectaría de forma adversa a la función renal residual.

Por lo tanto, la bioimpedancia es una herramienta útil, práctica y fácil de manejar que aporta una información objetiva para el seguimiento del paciente en programa de diálisis peritoneal. 


\section{Bioimpedance: a common tool in the care of peritoneal dialysis (PD) patients}

\section{Abstract}

The state of hydration of patients should be considered an indicator of adequate dialysis, as well as influencing directly on the patient's quality of life.

Bioimpedance is an objective method for nutritional and hydric monitoring and assessment of patients.

The regular measurements can be a useful aid to clinical assessment and the identification of variations in hydration and nutrition, allowing for suitable changes in conduct.

In our unit, we considered using bioimpedance as a tool to detect the state of hydration and the corporal composition of patients in order to draw up an individualized care plan.

A study was carried out at our centre analysing, in a sample of 8 patients on the peritoneal dialysis programme, the bioimpedance measurements routinely taken at each check-up.

The variables recorded were age, cause of renal disease (RD), weight, height, sex, time on dialysis, blood pressure (BP), glomerular filtration (GF), number of hypotensors, diuretics and results obtained from the bioimpedance measurements.

We can conclude that bioimpedance detects patients with non-volume-dependent hypertension. In this cases it is fundamental not to force the increase in UF to reduce the $\mathrm{BP}$, as this would have an adverse effect on residual renal function.

Accordingly, bioimpedance is a useful, practical and easy to use tool that contributes objective information for monitoring patients on peritoneal dialysis.

\section{KEY WORDS:}
- STATE OF HYDRATION
- CORPORAL COMPOSITION
- BIOIMPEDANCE
- HEALTH EDUCATION
- RESIDUAL RENAL FUNCTION

\section{Introducción}

De los diagnósticos de enfermería más prevalentes en los pacientes en programa de diálisis peritoneal (DP), podríamos destacar los siguientes:

- Desequilibrio nutricional.

- Desequilibrio de volumen de líquidos.

- Déficit de conocimientos sobre dieta.

Una de las funciones más importantes del tratamiento con diálisis es el correcto balance de líquidos.

La DP, al tratarse de una técnica continua resulta muy adecuada para conseguir un óptimo estado de hidratación, sin fluctuaciones y con mayor estabilidad hemodinámica, sin embargo esto no es siempre posible como lo demuestra la alta prevalencia de hipertensión arterial (HTA) y la alta mortalidad de causa cardiovascular.

El estado de hidratación de los pacientes debe ser considerado como un indicador de diálisis adecuada, además de influir directamente en la calidad de vida del paciente ${ }^{1}$.

La sobrehidratación se produce por una ingesta de líquidos superior a las pérdidas (ultrafiltración más diuresis residual). Es frecuente al perder la función renal residual. Cursa con edemas, elevación de la presión arterial, aumento de peso y, en casos graves, insuficiencia cardíaca. En su diagnóstico debe descartarse el déficit de ultrafiltración. Su profilaxis radica en preservar la función renal residual, el control de la ingesta de líquidos, dieta hiposódica y el uso adecuado de las concentraciones de glucosa, ajustando las permanencias al tipo de transporte peritoneal ${ }^{2}$.

La valoración de la distribución del volumen corporal a través de parámetros subjetivos (valoración de signos y síntomas), es un factor limitante en el control del peso seco.

El método de la impedancia bioeléctrica se basa en la conducción de una corriente de baja intensidad a través del cuerpo del paciente. La masa magra conduce la electricidad más fácilmente, ya que tiene un alto contenido de agua y electrólitos, mientras que la masa grasa opone más resistencia. Así se deduce que la masa magra conduce la corriente eléctrica con más facilidad que la masa grasa. 
Se trata de un método de valoración objetiva y de monitorización nutricional e hídrica de los pacientes en DP. Las mediciones periódicas pueden ser complemento útil para la valoración clínica y la identificación de variaciones en la hidratación y nutrición, permitiendo alteraciones adecuadas de la conducta. Su aplicación ideal no es la de la valoración puntual, sino la de una monitorización longitudinal del paciente. Es un método apropiado para la valoración de las alteraciones en la composición corporal durante el seguimiento del paciente ${ }^{3}$.

Poder conocer la composición corporal en los pacientes renales es una herramienta útil para la adecuación de los cuidados de enfermería en la consulta de DP.

La bioimpedancia es una herramienta fiable, objetiva y precisa. Se trata de una técnica no invasiva, de fácil ejecución, reproducible que requiere poco tiempo en su realización y que nos dará una medida objetiva de hidratación, para un ajuste preciso del peso seco en función de la situación clínica.

En nuestra unidad disponemos del monitor Body Composition Monitor (BCM) de Fresenius Medical Care. Este monitor emplea tecnología espectrOSCópica (BIS) y toma mediciones a 50 frecuencias comprendidas en un rango de 5 a $1.000 \mathrm{KHz}$, determinando el agua corporal total (TBW) y el agua extracelular $(E C W)^{4}$. Este monitor tiene la ventaja de que mide la sobrehidratación como dato cuantitativo en litros.

A través del monitor de bioimpedancia $B C M$, se pueden valorar los siguientes parámetros clínicos entre otros:

Cuantificación del estado de hidratación:

- Sobrehidratación (OH) (L).

- Agua corporal (V) (L).

- Volumen extracelular (ECW) (L).

Cálculo de la composición corporal:

- Masa de tejido magro (LTM) (Kg.) Con esta medida calculamos el índice de tejido magro (LTI=LTM/ altura ${ }^{2}$ ) para comparar con una población del mismo sexo y edad.

- Masa de tejido adiposo (ATM) (Kg.) Con esta medida calculamos el índice de tejido adiposo (FTI=ATM/altura ${ }^{2}$ ) para comparar con una población del mismo sexo y edad.

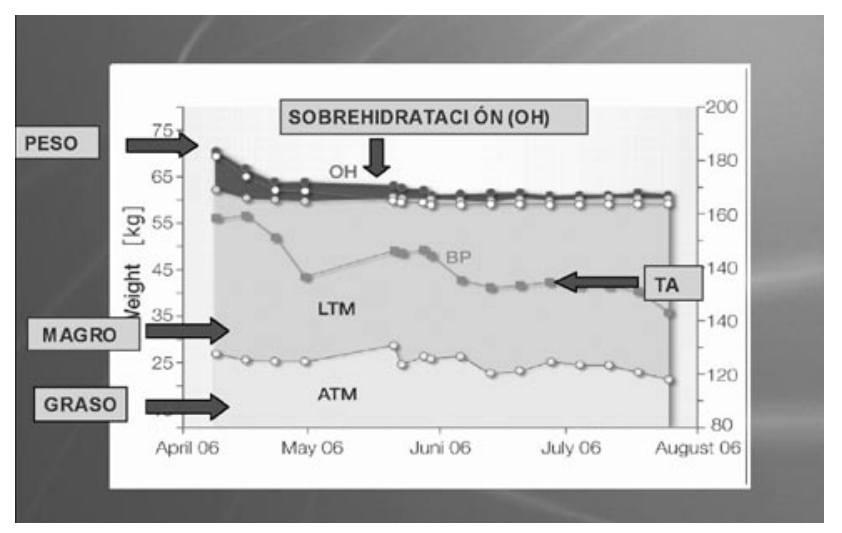

Figura 1. Diagrama de Composición corporal según BCM (Body Composition Monitor).

Toda esta información complementaria orientará la aplicación de los cuidados de enfermería, educación sanitaria y modificación de hábitos de nuestros pacientes, evitando la sobrehidratación y la preservación de la función renal residual.

Por tanto, en nuestra unidad de DP nos planteamos utilizar la bioimpedancia como herramienta para detectar el estado de hidratación y vigilar los cambios en la composición corporal y con ello elaborar el plan de cuidados individualizados de cada uno de nuestros pacientes.

\section{Objetivos}

- Describir el estado de hidratación.

- Describir el estado de la composición corporal.

\section{Material y métodos}

Se trata de un estudio descriptivo retrospectivo de las mediciones de bioimpedancia obtenidas mensualmente de los pacientes de una unidad de diálisis peritoneal.

Las mediciones de bioimpedancia se han realizado a través del monitor Body Composition Monitor (BCM) de Fresenius Medical Care.

Las mediciones del BCM se realizan siempre en las mismas condiciones; se coloca al paciente en decúbito supino sobre superficie no conductora en reposo 2 
minutos antes de la medición. Hay que comprobar que el paciente no es portador de objetos metálicos. Está contraindicado el uso en pacientes portadores de marcapasos o stent metálico. El paciente deberá, en la medida de lo posible, abrir ligeramente las piernas y los brazos.

Se debe limpiar la piel con alcohol el dorso de la mano y el empeine del pie del mismo lado antes de colocar los electrodos, eligiendo preferiblemente el lado no dominante del paciente. Si es potador de acceso vascular se elegirá el lado opuesto para realizar la medición.

Figura 2. Colocación de electrodos.

Antes de hacer la medida se tomará la presión arterial

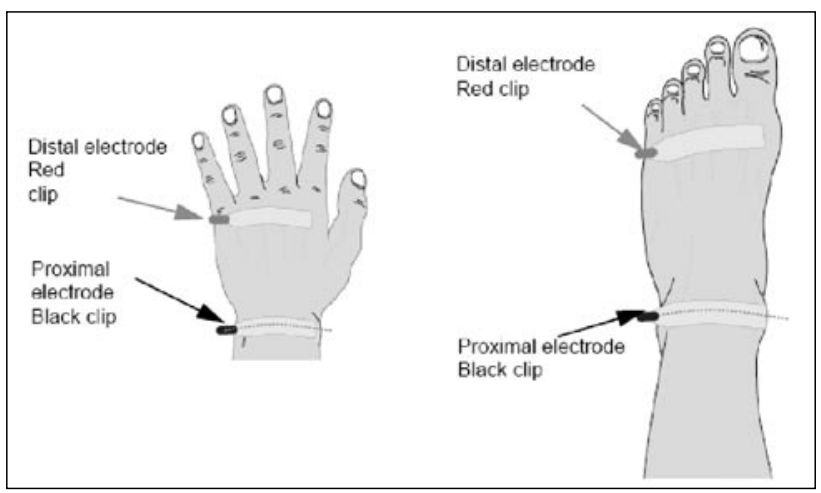

(PA). Ésta se ha obtenido a través del monitor de presión arterial Omron MI plus clínicamente validado. EI peso corporal se ha obtenido a través de una báscula de marca "SECA" calibrada.

La muestra la formaron todos los pacientes en programa de diálisis peritoneal que de forma rutinaria se realizaron una medición por bioimpedancia en cada revisión.

Las soluciones de DP utilizadas por los pacientes en el tiempo de estudio fueron en 6 de ellos Bicavera glucosa y en los 2 restantes CAPD2 stay-safe.

La medición se realizó generalmente con el peritoneo lleno y del peso corporal total se tuvo que restar el volumen que tuviera intraperitoneal. El volumen intraperitoneal no modifica el valor de la $\mathrm{OH}$, pero sí modifica el cálculo de la composición corporal (no se puede tomar como peso corporal).

Las variables recogidas fueron edad, causa de enfermedad renal (ER), peso, altura, sexo, tiempo en diálisis, Tensión Arterial, filtrado glomerular (FG), $n^{0}$ de hipo- tensores, diuréticos y resultados de la bioimpedancia $(\mathrm{OH}, \mathrm{ECW}, \mathrm{LTI}, \mathrm{FTI})$.

También se recogió el resultado del cociente $0 \mathrm{H} / \mathrm{ECW}$, considerando como valor de sobrehidratación alterado un valor de cociente de sobrehidratación en relación con el volumen extracelular $>15 \% 6$.

El cociente $\mathrm{OH} / \mathrm{ECW}$ se recogió entendiendo que no es de igual relevancia la sobrehidratación en función del peso corporal del paciente, es decir, hay una gran diferencia en una sobrehidratación de 5 litros en un paciente cuyo peso corporal sea $50 \mathrm{Kg}$ que en un paciente que pese $100 \mathrm{Kg}$.

Se ha considerado HTA aquella $\geq 140 / 90 \mathrm{mmHg} 5$, como indican las guías de HTA 2005.

Estos datos de la BCM se obtuvieron tras procesar la medición mediante el programa informático que adjunta el sistema, en el cual se comparan los resultados de $\mathbf{0 H}$, LTI y FTI con una población de referencia de igual sexo y edad. El valor 0 indica normalidad, el 1 un valor $>$ a lo normal y el -1 un valor < a lo normal.

\section{Resultados}

Se recogieron los datos de 8 pacientes; 3 mujeres y 5 hombres de edad media 44,85 (rango: 18-69 años). Los datos se recogieron desde marzo 2009 al marzo 2010, según se fueron incorporando al programa de diálisis, con una media de tres medidas/paciente.

\section{Las causas de ER eran:}

- Nefropatía diabética: 2 casos (25\%).

- Poliquistosis: 2 casos (25\%).

- Glomerulonefritis proliferativa: 1 caso $(12,5 \%)$.

- Enfermedad renal vascular por HTA: 1 caso (12,5\%).

- Desconocida: 2 casos (25\%).

El tiempo medio de estancia en programa de DP fue de 6.5 (rango 2-14 meses).

La modalidad de tratamiento ha ido variando en el tiempo de estudio, habitualmente comenzaron en DPCA, 4 pasaron a DPA y 1 tuvo que ser trasferido a HD

Se han realizado 24 mediciones en total y los resultados, expresados como media de cada paciente vienen expresados en la Tabla 1 y en la Tabla 2. 


\begin{tabular}{|c|c|c|c|c|c|c|c|c|c|c|c|}
\hline PTE & DIUR & FG & UF & $\mathrm{OH}$ & $\mathrm{OH}$ & ECW & OH/ECW & TAS & TAD & HIPOTA & FCOS DIUR \\
\hline & L & $\mathrm{ml} / \mathrm{min}$ & L & L & & L & $\%$ & $\mathrm{mmHg}$ & $\mathrm{mmHg}$ & $\mathrm{N}^{\circ}$ & $\mathrm{N}^{\circ}$ \\
\hline 1 & 1,775 & 4,4 & 0,42 & 9,2 & SI & 42,2 & 39,06 & 180,75 & 103,2 & 3,5 & 1 \\
\hline 2 & 2,46 & 19,31 & 0,4838 & 2,98 & SI & 46,42 & 13,06 & 125,6 & 73,8 & 4,2 & 1 \\
\hline 3 & 0,616 & 7,52 & 0,5866 & 2,12 & SI & 39,75 & 10,53 & 158,5 & 92,75 & 1,75 & 1 \\
\hline 4 & 2,675 & 16,5 & $-0,348$ & 0,4 & NO & 34,77 & 2,27 & 137,75 & 91,25 & 2 & 1 \\
\hline 5 & 1,06 & 9,75 & $-0,019$ & 2,6 & SI & 31,15 & 15,9 & 129,5 & 78,5 & 1,5 & 1 \\
\hline 6 & 1,425 & 11,15 & 1,52 & 9,55 & SI & 41,15 & 38,2 & 197,5 & 86 & 2 & 1 \\
\hline 7 & 0,55 & 5,35 & 0,445 & -1 & NO & 33,15 & $-6,65$ & 143 & 78,5 & 3 & 1 \\
\hline 8 & 1,325 & 2,63 & 0,0305 & 1,8 & SI & 26,05 & 14,6 & 112 & 57 & 0 & 0 \\
\hline
\end{tabular}

Tabla 1. Variables recogidas.

\begin{tabular}{|c|c|c|c|c|c|c|c|}
\hline PTE & Peso & IMC & ALB & LTI & DESV & FTI & DESV \\
\hline & $\mathrm{Kg}$ & $\mathrm{Kg} / \mathrm{m} 2$ & $\mathrm{~g} / \mathrm{dl}$ & $\mathrm{Kg} / \mathrm{m} 2$ & & $\mathrm{Kg} / \mathrm{m} 2$ & \\
\hline 1 & 72,9 & 22 & 3,05 & 12,15 & -1 & 7,45 & 1 \\
\hline 2 & 91,16 & 31 & 3,48 & 17,06 & 0 & 13 & 1 \\
\hline 3 & 75,8 & 24 & 3,8 & 14,2 & -1 & 9,1 & 0 \\
\hline 4 & 85,5 & 34 & 3,85 & 13,42 & 0 & 21,5 & 1 \\
\hline 5 & 73 & 26 & 4,05 & 10 & -1 & 16,05 & 1 \\
\hline 6 & 80,9 & 26 & 3,15 & 10,25 & -1 & 12,06 & 0 \\
\hline 7 & 87,9 & 32 & 4,15 & 11,1 & -1 & 21,5 & 1 \\
\hline 8 & 49,35 & 21 & 4 & 12,55 & 0 & 8,1 & 0 \\
\hline
\end{tabular}

Tabla 2. Variables recogidas.

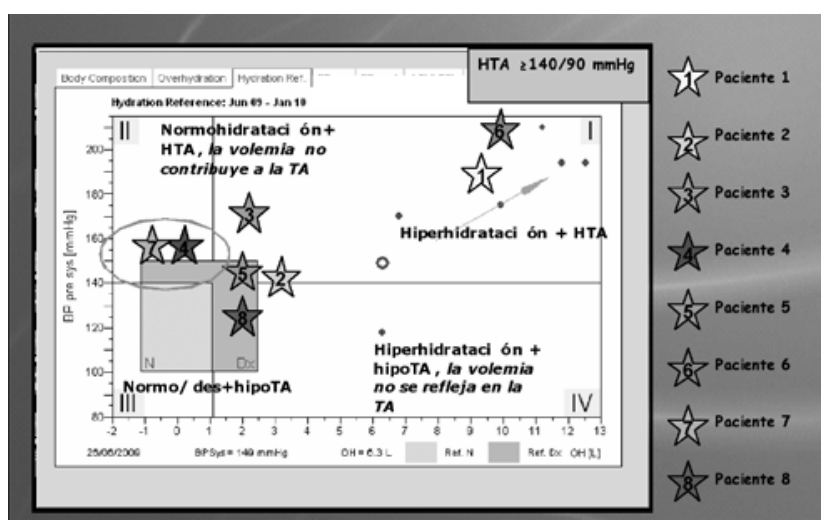

Gráfico 1. Estado de hidratación interpretada por OH y TAS (Tensión Arterial Sistólica).

Así observamos que del total de los pacientes, 5 de ellos están hipertensos y sólo 1 de ellos no toma medicación hipotensora ni diuréticos.

Con respecto al estado de hidratación interpretada por $\mathrm{OH}$, del total de los pacientes, 6 presentan sobrehidratación (> de 1 litro) y 2 normohidratación.

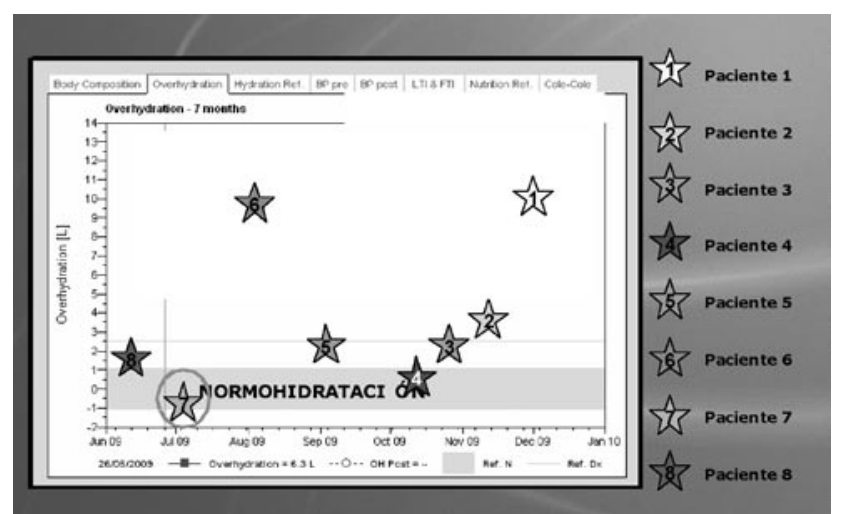

Gráfico 2. Estado de hidratación interpretada por $\mathrm{OH}$.

Si la sobrehidratación se interpreta por $0 \mathrm{H} / \mathrm{ECW}$, los pacientes con sobrehidratación ( $>15 \%$ ) son sólo 3 y dos de ellos presentaban edemas importantes. 


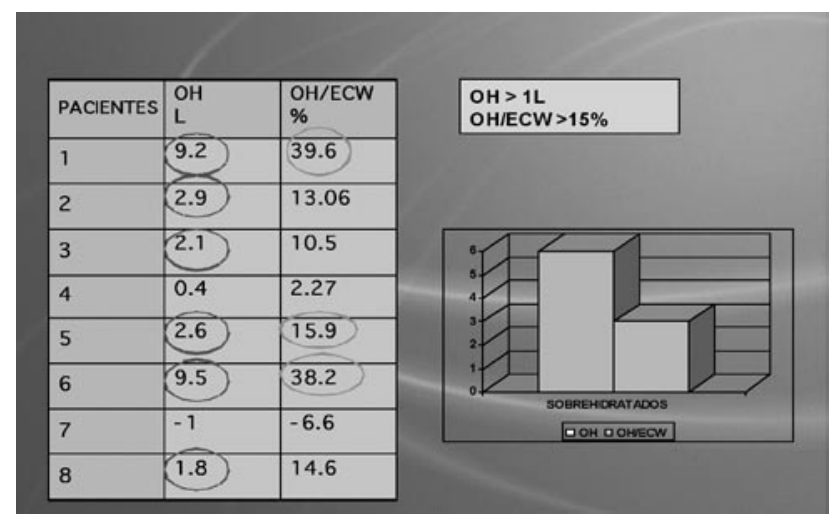

Gráfico 3. Estado de hidratación interpretada por $\mathrm{OH} / \mathrm{ECW}$ comparada con $\mathrm{OH}$.

- Pacientes con sobrehidratación interpretada por OH fueron 6:

- La media de $0 \mathrm{H}$ es de 4,7 litros.

- Sólo 3 de estos pacientes presentan HTA con una cifra media de 170/96 mm Hg.

- De los otros 3 pacientes con cifras de TA normales, 2 toman fármacos hipotensores y diuréticos y 1 no toma nada.

- 5 pacientes toman fármacos hipotensores, con una media de 2,45 fármacos hipotensores y 1 fármaco diurético.

- La diuresis media de estos pacientes es de 1,44 litros y el $\mathrm{FG}:$ 9,12 $\mathrm{ml} / \mathrm{min}$.

- La UF de diálisis peritoneal media es 0,5 litros.

- Los pacientes con mayor sobrehidratación (media de 9,3 litros), son los que a su vez presentan menores cifras de albúmina sérica $(3,1 \mathrm{~g} / \mathrm{dl})$.

- Pacientes con normohidratación fueron 2:

- La media de $0 \mathrm{H}$ es de 0,3 litros.

- Los 2 pacientes presentan HTA con una cifra media de 143/91 mmHg.

- Todos ellos toman fármacos hipotensores, con una media de 2,5 fármacos hipotensores y 1 fármaco diurético.

- La diuresis media de estos pacientes es de 1,612 litros y el FG: 10,9 $\mathrm{ml} / \mathrm{min}$.

- La Ultrafiltración (UF) de diálisis peritoneal media es 0,39 litros.

Con respecto a la composición corporal, del total de los pacientes, 5 presentan un índice de tejido magro por debajo de lo normal y 5 de ellos un índice de tejido

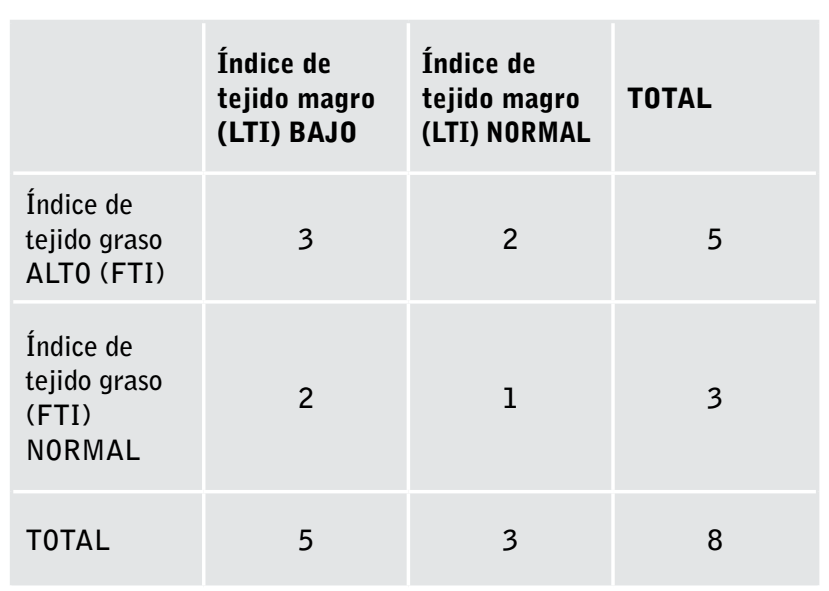

Gráfico 4. Resultados composición corporal.

adiposo por encima de lo normal.

En resumen, de nuestros pacientes, 5 presentan LTI bajo de los que 3 tenían exceso de grasa y 2 grasa normal; 3 tenían un LTI normal, de los que 2 tenían > grasa corporal y 1 normal.

En cuanto a la relación entre el Índice de masa corporal (IMC), el tejido magro y el tejido graso destacamos uno de los pacientes, que a pesar de tener IMC normal, presenta el índice de tejido graso elevado. Lo que confirma la importancia de la determinación de

\begin{tabular}{|c|c|c|c|c|c|}
\hline PACIENTES & PESO Kgr & IMC Kgr/m & ALBUMI & LTI & FTI \\
\hline 1 & 72.9 & 22 & 3.05 & & \\
\hline 2 & 91.1 & 31 & 3.48 & & \\
\hline 3 & 75.8 & 24 & 3.8 & & \\
\hline 4 & 85.5 & 34 & 3.85 & & \\
\hline 5 & 73 & 26 & 4.05 & & \\
\hline 6 & 80.9 & 26 & 3.15 & & \\
\hline 7 & 87.9 & 32 & 4.15 & & \\
\hline 8 & 49.3 & 21 & 4 & & \\
\hline
\end{tabular}

Gráfico 5. Composición corporal. Relación entre IMC/ FTI/LTI.

la grasa y la masa muscular sobre el IMC.

\section{Discusión y conclusiones}

El presente trabajo describe la utilidad práctica del uso de BCM como herramienta para mejorar el cuidado 
de los pacientes en DP.

- Para la evaluación del estado de hidratación lo principal es la valoración integral del paciente. El OH aportará información adicional para individualizar los cuidados de enfermería.

- El cociente $0 \mathrm{H} / \mathrm{ECW}$ se entiende como un factor predictivo de mortalidad.

- La BIE (Bioimpedancia Eléctrica) detecta pacientes con HTA no dependiente de volumen. En estos casos es fundamental no forzar el aumento de UF para disminuir la PA, puesto que esto afectaría de forma adversa a la función renal residual.

- Las intervenciones de enfermería irán orientadas al manejo óptimo de los líquidos y recomendaciones dietéticas para disminuir la ingesta de sal.

- La mayor parte de los pacientes tienen un contenido de tejido graso elevado y de magro disminuido. Nuestro objetivo educacional irá orientado a mejorar esta situación y disminuir el riesgo cardiovascular, a través de la promoción de hábitos de vida saludables y ejercicio físico adecuado.

- La BIE es una herramienta útil, práctica y fácil de manejar que aporta una información objetiva para el seguimiento del paciente en programa de DP.

\section{Limitaciones}

- Muestra pequeña.

- Tiempo corto de seguimiento para valorar resultados de intervención.

- Nuestro objetivo es continuar con este estudio, ampliándolo.

\section{Recibido: Marzo 2011}

Revisado: Abril 2011

Modificado: Mayo 2011

Aceptado: Julio 2011

\section{Bibliografía:}

1. Remón-Rodríguez C, Miguel A, Pérez-Bañasco V, Caravaca F. Problemas de ultrafiltración y en el balance de líquidos en el paciente en diálisis peritoneal. Guías de diálisis peritoneal. SEN 8: 161-179, 2005.

2. Tornero F, Rivera M. Complicaciones no infecciosas del paciente en la diálisis peritoneal. En: Lorenzo V, López JM, de Francisco ALM, Marrero D. Nefrología al día. Barcelona; Plus medical 2010:36: Pag. 615-616.

3. Riella M, Massaki M, Carreira S. La nutrición en diálisis peritoneal. En: Montenegro J, Correa-Rotter $\mathrm{R}$, Riella M. Tratado de diálisis peritoneal. Barcelona; Elseiver 2009: 26: 497.

4. Fresenius Medical Care. Body Composition Monitor [acceso 28 julio 2011] Disponible en: http://www. bcm-fresenius.com/index.html.

5. Martín R. Guía española de hipertensión 2005. SEH-LELHA. Hipertensión. 2005; 22 Supl 2:3-8.

6. Devolver I, Verleysen A, Vijt D, Vanholder R, Van Biesen W. Body composition, hydratation, and related parameters in hemodyalisis versus peritoneal dialysis patient. Perit Dial Int 2010; 30: 208-214. 